\title{
A NUMERICALLY EFFICIENT FINITE ELEMENT
}

\author{
HYDROELASTIC ANALYSIS * \\ Robert N. Coppolino \\ The Aerospace Corporation
}

\section{SUMMAR Y}

\begin{abstract}
A finite element hydroelastic analysis formulation is developed on the basis of Toupin's complementary variational principle. Emphasis is placed on the special case of an incompressible fluid model which is applicable to propellant tank hydroelastic analysis. A concise fluid inertia representation results from the assumption of incompressibility and the hydroelastic equations reduce to a simplified form associated with the structure alone. The efficiency of the incompressible hydroelastic formulation is enhanced for both fluid and structure by introduction of harmonic reduction as an alternative to Guyan reduction. The theoretical developments are impelemented in the NASTRAN Program and the technique is verified and demonstrated as an efficient and accurate approach with a series of illustrative problems including the 1/8scale Space Shuttle external tank.
\end{abstract}

\section{INTRODUCTION}

The increasing complexity of launch vehicle configurations, particularly in the case of the Space Shuttle, recently has stimulated considerable interest in the dynamic behavior of liquid-filled tanks. The prevention of coupled structure-propulsion instability (pogo), for example, requires very complete and accurate mathematical models for the calculation of propellant tank hydroelastic modes (ref. 1) in the frequency range of concern $(2-50 \mathrm{~Hz}$ for the Space Shuttle).

Various fluid modeling techniques for hydroelastic analysis (refs. 2-7) have been mechanized for digital computation. These techniques range from finite element and finite difference techniques to approximate analytical approaches taking advantage of the properties of the fluid velocity potential and the consequences of Green's theorem. Most hydroelastic analysis methods, however, are limited to special geometric configurations. Furthermore, although theoretically rigorous, most contain deficiencies in computational economy and/or numerical accuracy.

\footnotetext{
*Work performed at Grumman Aerospace Corporation under Contracts NAS1 10635-21 and POM3WXMZ-483002 for NASA Langley Research Center and Rockwell International Space Division, respectively. $* *$

Member Technical Staff, Vehicle Engineering Division
} 
In particular, the hydroelastic analysis technique used in NASTRAN (ref. 7) is deficient in computational economy. This technique employs a large unsymmetrical eigenvalue problem formulated in terms of mixed fluid pressure and structural displacement generalized coordinates. In this formulation, the fluid coefficient matrices, derived by a Galerkin-type approach, are interpreted according to a structural analogy. The resulting fluid pseudomass and pseudo-stiffness matrices are recognized herein as flexibility and inverse mass (inertance) matrices, respectively, on the basis of Toupin's complementary variational principle (ref. 8). This revised interpretation is central to the formulation of the hydroelastic problem presented here. Although the effort in the present work was initially directed towards alleviation of difficulties encountered in the NASTRAN hydroelastic analysis, it has been found that Toupin's principle provides fundamental physical insight for a variational approach to hydroelasticity and it provides a rigorous basis for the development of fluid finite elements.

A derivation of fluid matrix equations on the basis of Toupin's principle, and manipulation of the interacting structure equations to a form consistent with the complementary principle, ultimately results in a symmetric kinetic formulation for compressible hydroelasticity. A detailed development of the compressible formulation is not presented in this paper. The special case of incompressible hydroelasticity is of primary interest as it is particularly applicable in the study of propellant tank dynamics.

In the special case of incompressibility, the interior fluid pressure fluctuations are algebraically dependent on surface pressure fluctuations. Although this property is illustrated from the viewpoint of a matrix approach, it is well known as a consequence of the application of Green's theorem to a continuum potential description. Moreover, the surface pressure fluctuations are algebraically related to the fluid accelerations normal to the bounding surface, and this is equivalent to a statement of the "surface flux" boundary condition. In addition, it is recognized on physical as well as mathematical grounds that there exists an overspecification in the surface pressure/acceleration relationships which implicitly defines a compatibility condition. In very simple terms the condition which requires that under uniform pressure the fluid will not move manifests itself as a necessary singularity in the fluid inertance matrix. Upon elimination of the surface pressure singularity by introduction of pressure deviation dynamic variables, a concise fluid mass matrix is formed in terms of bounding-surface displacements alone; this matrix is simply added to the structural mass matrix, resulting in a symmetric kinematic set of hydroelastic dynamic equations. This description represents a drastic reduction in system variables.

It is noted in this paper that the sequencing of kinematic reduction operations (e.g., Guyan, etc.) on fluid and structure is crucial to the economy of the analysis approach. In addition, a matrix harmonic reduction scheme is introduced as an efficient alternative to Guyan reduction for geometrically, but not necessarily structurally, axisymmetric configurations to further reduce the number of system variables.

A series of illustrative problems is presented to demonstrate the present incompressible hydroelastic analysis, which has been implemented in 
NASTRAN. In particular the problems illustrate the use of harmonic reduction, and the accuracy and efficiency of the hydroelastic formulation in general, by comparison with exact analytical and available test results. Moreover, computation times for the standard NASTRAN and the present hydroelastic analysis techniques are compared.

\section{SYMBOLS}

A

B

C

$\mathrm{C}_{\mathrm{f}}, \mathrm{C}_{\mathrm{s}}$

E

$F_{\text {s }}$

G

I

K

$\mathrm{K}_{\mathrm{s}}, \mathrm{K}_{\mathrm{s}}^{*}$

L

$\mathrm{L}_{\mathrm{f}}, \mathrm{L}_{\mathrm{s}}$

$\mathrm{M}_{\mathrm{f}}$

$M_{s}, M_{s}^{*}$

$\mathrm{M}_{\theta}, \mathrm{M}_{\mathrm{z}}$

$\mathrm{N}_{\theta}, \mathrm{N}_{\mathrm{z}}$

$P, P^{\prime}$

R

S

$\mathrm{T}_{\mathrm{c}}, \mathrm{U}_{\mathrm{c}}$ generalized area matrix

fluid bulk modulus

flexibility matrix

fluid, structural flexibility matrix

structural elastic modulus

internal structural generalized force

matrix defined in equation (29b)

identity matrix

stiffness matrix

structural stiffness matrix, constrained structural stiffness matrix [eq. (32d)]

inertance matrix

fluid, structural inertance matrix

fluid mass matrix

structural mass matrix, constrained structural mass matrix [eq. $(32 \mathrm{c})]$

cylindrical-shell bending-moment resultants [eq. (40a)]

cylindrical-shell membrane-stress resultants [eq. (40a)]

pressure, pressure deviation array

hemisphere or cylindrical shell radius

surface area

complementary kinetic, potential energy function 


\begin{tabular}{|c|c|}
\hline$T_{s d}, T_{h}$ & $\begin{array}{l}\text { reduction transformation [eq. (34)], harmonic tr } \\
\text { [eq. (36)] }\end{array}$ \\
\hline $\mathrm{U}$ & displacement array \\
\hline $\mathrm{V}$ & volume \\
\hline$\delta W_{c}$ & complementary virtual work function \\
\hline h & shell thickness \\
\hline$\ell$ & cylindrical shell axial dimension \\
\hline $\mathrm{m}, \mathrm{n}$ & meridional, circumferential wave index \\
\hline$\hat{\mathbf{n}}$ & surface outward normal unit vector \\
\hline $\mathrm{p}$ & pressure \\
\hline $\mathrm{p}_{\mathrm{o}}$ & static pressurization level (gage) \\
\hline $\begin{array}{l}\mathrm{p}_{\mathrm{o}}\left(\mathrm{r}_{\mathrm{i}}, \mathrm{z}_{\mathrm{i}}\right), \\
\mathrm{p}_{\mathrm{K}}\left(\mathrm{r}_{\mathrm{i}}, \mathrm{z}_{\mathrm{i}}\right), \\
\mathrm{p}_{\mathrm{K}}^{*}\left(\mathrm{r}_{\mathrm{i}}, z_{i}\right)\end{array}$ & $\begin{array}{l}\text { harmonic distribution pressure amplitudes } \\
\text { [eq. (37)] }\end{array}$ \\
\hline $\mathbf{r}$ & radial coordinate in cylindrical reference frame \\
\hline $\mathbf{t}$ & time \\
\hline $\mathrm{u}, \overrightarrow{\mathrm{u}}$ & displacement, displacement vector \\
\hline $\mathbf{u}^{*}$ & surface displacement \\
\hline $\mathbf{z}$ & axial coordinate in cylindrical reference frame \\
\hline$\Gamma$ & matrix defined in equation $(28 c)$ \\
\hline$\Omega$ & nondimensional frequency \\
\hline$a$ & stiffness constant for hemis phere [eq. (38)] \\
\hline$\nu$ & Poisson's ratio \\
\hline$P_{f}, P_{s}$ & fluid, structural density \\
\hline$\omega$ & circular frequency \\
\hline
\end{tabular}


d( ) total differential

$\nabla \cdot() \quad$ divergence

$\nabla($ ) gradient

$\partial($ ) partial derivative

$\delta($ variation

$(1)$

total impulse $\int_{-\infty}^{t}() d t$

(') time derivative, $\mathrm{d}(\mathrm{)} / \mathrm{dt}$

Subscripts

$i, j, k$ denote elements of a matrix or vector

Abbreviations

DOF

degrees of freedom

CPU

central processing unit

\section{THEORETICAL DEVELOPMENT}

The class of problems considered here consists of the interaction of irrotational, inviscid, compressible fluids with flexible structures for which both fluid and structural motions are as sumed small compared to overall dimensions. The approach used to describe the dynamics of the fluid is a finite element technique which utilizes energy expressions based on Toupin's principle (ref. 8). A detailed derivation of Toupin's principle and a complementary form of Hamilton's principle are presented in reference 9 as consequences of a postulated complementary $D^{\prime}$ Alembert principle.

The equation of motion of a fluid particle is

$$
\ddot{\vec{u}}=-\frac{1}{p_{f}} \nabla p
$$

and the constitutive relationship for an inviscid, compressible fluid is

$$
\mathrm{p}=-\mathrm{B} \nabla \cdot \overrightarrow{\mathrm{u}}
$$


where $\nabla \cdot \vec{u}$ represents the dilatational strain. In order to obtain a fluid velocity expression, equation (1) is integrated to yield

$$
\dot{\overrightarrow{\mathrm{u}}}=-\frac{1}{\rho_{\mathrm{f}}} \nabla \hat{\mathrm{p}}
$$

where $\hat{\mathrm{p}}$ is the pressure impulse

$$
\hat{\mathrm{p}}=\int_{-\infty}^{t} \mathrm{pdt} \quad \text { or } \quad \dot{\hat{p}}=\mathrm{p}
$$

Complementary kinetic and strain energy ( $T_{c}$ and $U_{c}$, respectively) may now be expressed in terms of impulsive pressure (the complementary dynamic variable) as

$$
\begin{aligned}
& T_{c}=\frac{1}{2} \int_{V} \frac{1}{\rho_{f}}(\nabla \hat{p} \cdot \nabla \hat{p}) d V \\
& U_{c}=\frac{1}{2} \int_{V} \frac{1}{B}(\dot{\hat{p}})^{2} d V
\end{aligned}
$$

The motion dependent and impulse dependent energy expressions are generally not equivalent; they are equivalent, however, for linear systems (ref. 10). The complementary virtual work performed by boundary surface displace-
ments, $\vec{u}^{*}$, is

$$
\delta W_{c}=\int_{S} \delta \hat{p}(\dot{\vec{u}} * \hat{n}) d S
$$

The concept of complementary virtual work (ref. 10) may be viewed as a consequence of a complementary D'Alembert principle (ref. 9).

The above energy expressions substituted into the complementary form of Hamilton's principle

$$
\delta \int_{t_{0}}^{t_{1}}\left(T_{c}-U_{c}\right) d t+\int_{t_{0}}^{t_{1}} \delta W_{c} d t=0
$$


ultimately yields, after use of Green's theorem and integration by parts, integrated expressions corresponding to the wave equation and natural boundary conditions.

The complementary formulation is presently applied, however, as an approximate analysis tool. Consider an approximation of a fluid pressure (impulse) state expressed as a linear function of a finite set of generalized impulses. Let us also require that any such approximation contains spatially uniform pressure as a state describable by the chosen generalized impulses; this is analogous to the requirement in kinematic finite element analys is that assumed displacement states must contain rigid-body motions. The fluid complementary kinetic and strain energies resulting from the assumed pressure impulse states are therefore the quadratic functions

$$
\begin{aligned}
& T_{c}=\frac{1}{2} \sum_{i} \sum_{j} L_{i j} \hat{p}_{i} \hat{p}_{j} \\
& U_{c}=\frac{1}{2} \sum_{i} \sum_{j} C_{i j} \dot{\hat{p}}_{i} \dot{\hat{p}}
\end{aligned}
$$

with the elements of the symmetric inertance matrix $L$ and flexibility matrix C defined as

$$
L_{i j}=\frac{\partial^{2} T_{c}}{\partial \hat{p}_{i} \partial \hat{p}_{j}}, C_{i j}=\frac{\partial^{2} U_{c}}{\partial \hat{p}_{i} \partial \hat{p}_{j}}
$$

The $L_{i j}$ are proportional to $1 / \rho_{f}$ and the $C_{i j}$ are proportional to $1 / B$. The complementary virtual work is expressed as

$$
\delta \mathrm{W}_{c}=\sum_{i} \int_{S}\left[\frac{\partial \hat{\mathrm{p}}}{\partial \hat{\mathrm{p}}_{i}} \dot{\mathrm{u}}^{*} \cdot \hat{\mathrm{n}}\right] \mathrm{dS} \delta \hat{\mathrm{p}}_{\mathrm{i}}
$$

For the special case in which the surface displacements are physically dis cretized, the complementary virtual work may be expressed as

$$
\delta W_{c}=\sum_{k} \sum_{i} A_{i k} \dot{u}_{k}^{*} \delta \hat{p}_{i}
$$


with an element of the generalized area matrix A defined as

$$
A_{i k}=\int_{S_{k}}\left(\frac{\partial \hat{p}}{\partial \hat{p}_{i}}\right)\left[\frac{\partial \dot{\vec{u}}^{*}}{\partial \dot{u}_{k}^{*}} \cdot \hat{n}\right] d S
$$

Substitution of equations (8) and (11) into equation (7), with the appropriate integrations by parts, results in the complementary Euler-Lagrange equations

$$
\sum_{j}\left(L_{i j} \hat{p}_{j}+C_{i j} \ddot{\hat{p}}_{j}\right)=-\sum_{k} A_{i k} \dot{u}_{k}^{*}
$$

By taking the time derivative of this expression noting equation (4), the EulerLagrange equations become

$$
\sum_{j}\left(L_{i j} p_{j}+C_{i j} \ddot{p}_{j}\right)=-\sum_{k} A_{i k} \ddot{u}_{k}^{*}
$$

This is the form of the fluid dynamic finite element equations for individual elements and stacked systems of elements. In the case of a stacked system of elements, the matrix A represents only bounding surface generalized areas and $u_{k}^{*}$ represents discrete surface displacements. The pressures $\mathrm{p}_{j}$ comprise the set of boundary surface and internal pressures; therefore the matrix $A$ is rectangular.

The above set of fluid equations is derived for NASTRAN (ref. 7) by a Galerkin-type approach which "constructs" a minimal principle. In this latter approach, the matrix expressions are interpreted according to a mathematical analogy and physical insight is lost; that is, $L_{i j}$ is mathematically analogous to kinematic stiffness, $C_{i j}$ is analogous to kinematic mass and the right-hand side is analogous to a kinematic generalized force vector.

\section{A Symmetric Kinetic Formulation for Compressible Hydroelasticity}

In the general case of compressible fluid/structure interaction, the fluid is described in terms of the complementary form

$$
L_{f} P+C_{f} \ddot{P}=-A^{T} \ddot{U}
$$


and the structure under fluid pressure excitation is described in the standard kinematic form

$$
M_{s} \ddot{U}+K_{s} U=A P
$$

Direct coupling of the above set in terms of the mixed pressure and structural displacement variables [as in the standard NASTRAN formulation (eq. (7))] results in an unsymmetric eigenvalue problem.

A symmetric formulation can be derived by the complementary principle or by an equivalent manipulation of the structural dynamic equations to the complementary form. If the latter approach is taken, internal structural generalized forces, $F_{S}$, are related to the structural displacements, $U$, according to

$$
\mathrm{K}_{\mathbf{S}} \mathrm{U}=\mathrm{F}_{\mathbf{S}}
$$

Suppose that $\mathrm{K}_{\mathrm{s}}$ represents a stiffness matrix of an inter acting supported structure such that rigid body deflections do not occur; the transformation to inter nal forces is defined as

$$
\mathrm{U}=\mathrm{K}_{\mathrm{S}}^{-1} \mathrm{~F}_{\mathrm{S}}
$$

Incorporation of this transformation into the hydroelastic equation set, equation (14), yields the symmetric set of hydroelastic equations in terms of forcetype variables.

$$
\left[\begin{array}{c:c}
\mathrm{C}_{\mathrm{f}} & 0 \\
\hdashline 0 & \mathrm{C}_{\mathbf{s}}
\end{array}\right]\left\{\begin{array}{l}
\ddot{\mathrm{P}} \\
\hdashline \ddot{\mathrm{F}}_{\mathrm{s}}
\end{array}\right]+\left[\begin{array}{c:c}
\mathrm{L}_{\mathrm{f}}+\mathrm{A}_{\mathrm{L}} \mathrm{A} & -\mathrm{A}_{\mathrm{s}}^{\mathrm{T}_{\mathrm{s}}} \\
\hdashline-\mathrm{L}_{\mathbf{s}} \mathrm{A} & \mathrm{L}_{\mathrm{s}}
\end{array}\right]\left\{\begin{array}{c}
\mathrm{P} \\
\hdashline \mathrm{F}_{\mathbf{s}}
\end{array}\right\}=\left\{\begin{array}{c}
0 \\
\hdashline 0
\end{array}\right\}
$$

with the structural inertance and compliance matrices defined, respectively, as

$$
\mathrm{L}_{\mathrm{s}}=\mathrm{M}_{\mathrm{s}}^{-1}, \mathrm{C}_{\mathrm{s}}=\mathrm{K}_{\mathrm{s}}^{-1}
$$

The formulation presented here provides a symmetric kinetic formulation for inviscid, compressible fluid hydroelastic problems for which efficient eigenvalue analysis techniques are applicable. Further discussion of the compressible formulation is not presently warranted since the class of problems of interest is limited to incompressible fluids interacting with flexible structures. The alternate simplified kinematic formulation to be derived below is appropriate for this case. 
In the special case for which fluid compressibility is negligible $\left(C_{f} \sim 0\right)$ the complementary fluid dynamic equations, equation (14a), reduce to a set of algebraic equations relating fluid pressures and boundary-surface accelerations. One is strongly motivated to utilize this quality to solve for the pres sures in terms of surface accelerations --and ultimately obtain a kinematic formulation which is identical in form to a standard set of structural dynamic equations.

Two fundamental properties of the fluid are recognized as consequences of the simplifying assumption of fluid incompressibility. These properties are necessarily inherent in both the continuum and the present matrix finite element descriptions. The first property requires that for an incompressible fluid the interior pressures are related to the surface pressures in a purely geometric sense; from the continuum viewpoint, this is a consequence of Green's theorem applied to an incompressible fluid. The second property requires that the net flux (or normal flow) out of the fluid volume is zero. This latter property amounts to a statement of constraint on flow normal to the bounding surface, and simultaneously it is recognized as a compatibility constraint on surface pressures. A simple physical statement of this second property is that, under the uniform surface pressure (impulse) state, the fluid surface will not deform and consequently, the fluid volume will not move. This point is illustrated mathematically by noting that in the uniform pressure state the pressure gradient, $\nabla \mathrm{p}$, is null throughout the fluid volume; hence the complementary kinetic energy, equation (5a), is null.

The complementary fluid matrix equation set for the special case of an incompressible fluid [see equation (14a) for $C_{f}=0$ ] in a conveniently partitioned form is expressed as

$$
\left[\begin{array}{ccc}
L_{f f} & L_{f s} & L_{f i} \\
L_{s f} & L_{s s} & L_{s i} \\
L_{i f} & L_{i s} & L_{i i}
\end{array}\right]\left\{\begin{array}{c}
P_{f} \\
P_{s} \\
P_{i}
\end{array}\right\}=-\left[\begin{array}{cc}
A_{f f}^{T} & A_{s f}^{T} \\
A_{f s}^{T} & A_{s s}^{T} \\
0 & 0
\end{array}\right] \mid \begin{gathered}
\ddot{U}_{f} \\
\ddot{U}_{s}
\end{gathered}
$$

The pressure partitions, $P_{f}, P_{s}$ and $P_{i}$ correspond to a single surface reference pressure or free-surface pressure set, the complement of the total surface pressure set, and the internal fluid pressure set, respectively; the displacement partitions $\mathrm{U}_{\mathrm{f}}$ and $\mathrm{U}_{\mathrm{S}}$ correspond to a single surface reference displacement or free surface displacement set and the complement of the total surface displacement set, respectively. The structural dynamic equation set with applied fluid pressure loading is, in partitioned form 


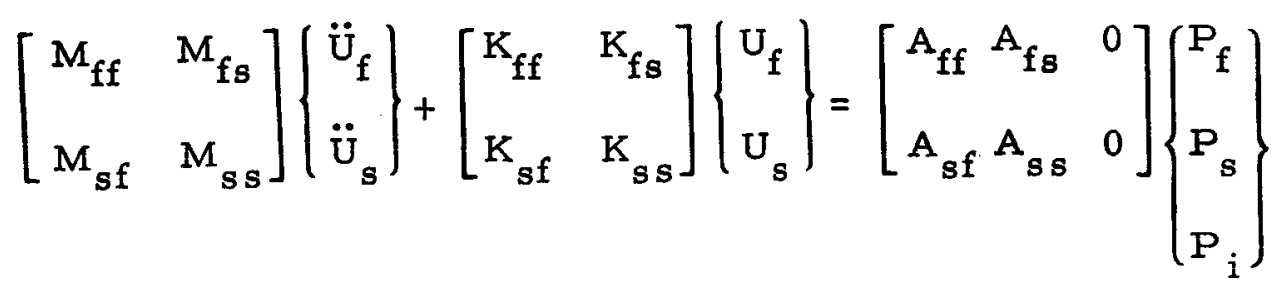

where the structural mass and stiffness matrices are expressed in partitioned form in accordance with the surface reference displacement set and the complement of the total surface displacement set, respectively. If the reference subsets of pressure, $P_{f}$, and displacement, $U_{f}$, should correspond to a free fluid surface rather than a structural interface, then the structural titions $K_{f f}, K_{f s}$, $K_{s f}$ would correspond to contributions due to the surface gravitational potential and possibly ullage pressure fluctuation. In many cases the free surface and ullage stiffness are negligible relative to the structural stiffness, $\mathrm{K}_{\mathrm{s}}$, and may therefore be neglected. The development of the incompressible hydroelastic equations presented below treats the most general case implied in equation (20), with the above special cases illustrated at the completion of the derivation.

From equation (19), the internal pressures are related to the surface pressures by

$$
P_{i}=-L_{i i}^{-1}\left[L_{i f} L_{i s}\right]\left\{\begin{array}{l}
P_{f} \\
P_{s}
\end{array}\right\}
$$

and the reduced fluid dynamic equation set, in terms of surface quantities only, is

$$
\left[\begin{array}{cc}
L_{f f}^{\prime} & L_{f s}^{\prime} \\
L_{s f}^{\prime} & L_{s s}^{\prime}
\end{array}\right]\left\{\begin{array}{c}
P_{f} \\
P_{s}
\end{array}\right\}=-\left[\begin{array}{cc}
A_{f f}^{T} & A_{s f}^{T} \\
A_{f s}^{T} & A_{s s}^{T}
\end{array}\right]\left\{\begin{array}{c}
\ddot{U}_{f} \\
\ddot{U}_{s}
\end{array}\right\}
$$

with

$$
\left[\begin{array}{cc}
L_{f f}^{\prime} & L_{f s}^{\prime} \\
L_{s f}^{\prime} & L_{s s}^{\prime}
\end{array}\right]=\left[\begin{array}{ll}
L_{f f} & L_{f s} \\
L_{s f} & L_{s s}
\end{array}\right]-\left[\begin{array}{l}
L_{f i} \\
L_{s i}
\end{array}\right] L_{i i}^{-1}\left[L_{i f} L_{i s}\right]
$$


The reduced inertance matrix is singular since an incompressible fluid under uniform pressure does not deform. This singularity is assured in the individual finite element inertance matrices by the use of admissible pressure distributions; it is a necessary condition for compatibility.

For a fluid represented by discrete surface pressures the normalized uniform pressure state is

$$
\left\{\begin{array}{c}
P_{f} \\
-- \\
P_{s}
\end{array}\right\}=\left\{\begin{array}{c}
1 \\
-\frac{1}{i} \\
i
\end{array}\right\}
$$

Under such loading the surface normal accelerations must be null and the necessary property of the reduced inertance matrix is

$$
\sum_{j} L_{i j}^{\prime}=0
$$

The uniform pressure singularity pertains as well to the full pressure set with the members of $P_{i}$ also unity; the reduction to the surface pressure set is made in this derivation mainly to illustrate the first basic property, of geometric dependence of internal pressure. For axisymmetric fluid elements with generalized pressure variables corresponding to amplitudes of circumferential harmonics, this discussion pertains to the zeroth harmonic, only, which contains the uniform pressure singularity. The singularity is removable by introduction of the concept of pressure deviation in which the deviations from the reference uniform pressure state of value, $P_{f}$, are

$$
P_{s_{i}}^{\prime}=P_{s_{i}}-P_{f}
$$

Thus the relationship between pressures and pressure deviations may be expressed by the transformation

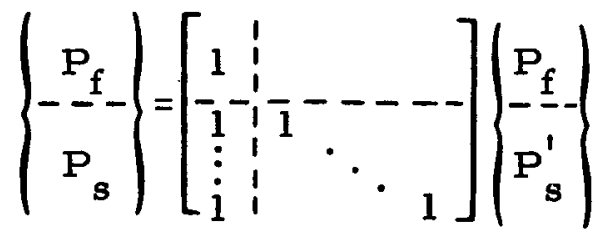

when $P_{f}$ represents a single discrete reference pressure. If $P_{f}$ represents a pressure subset comprised of all free surface pressures (to be nulled in the case of negligible surface gravitational potential and ullage pressure strain energy), the constraint relationship is somewhat different; consideration of this latter case, however, is reserved for a later part of the present discussion. 
Application of the above transformation on the reduced fluid equation set in equation (22), noting equation (24), yields

$$
\begin{aligned}
& L_{f s}^{\prime} P_{s}^{\prime}=-A_{f f}^{T} \ddot{U}_{f}-A_{s f}^{T} \ddot{U}_{s} \\
& L_{s s}^{\prime} P_{s}^{\prime}=-A_{f s}^{T} \ddot{U}_{f}-A_{s s}^{T} \ddot{U}_{s}
\end{aligned}
$$

Suppose now that the displacement $U_{f}$ is directed normal to the reference sur face; the area coupling partitions $A_{f_{s}}$ and $A_{S f}$ are therefore null, simplifying the above expressions. It should be noted that this restriction is made for clarity of the present development and the more general case is derivable with some additional algebraic complication. Solution for the pressure deviations in equation (27b) and substitution of the result in equation (27a) yields the pressure deviation recovery relationship and the displacement recovery relationship, respectively.

$$
\begin{gathered}
P_{s}^{\prime}=-L_{s s}^{\prime} A_{s s}^{T} \ddot{U}_{s} \\
{\left[\begin{array}{l}
U_{f} \\
U_{s}
\end{array}\left|=\left[\begin{array}{l}
\Gamma \\
I
\end{array}\right]\right| U_{s} \mid\right.}
\end{gathered}
$$

with

$$
\Gamma=A_{f f}^{-T} L_{f s}^{\prime} L_{s s}^{\prime-1} A_{s s}^{T}
$$

The latter result is equivalent to imposing a kinematic constraint on outward normal surface flow (incompressibility)

$$
\left\{\begin{array}{cc}
0 \\
A_{\text {s s }}^{T} & U_{s}
\end{array}\right\}=\left[\begin{array}{ll}
I & G \\
0 & I
\end{array}\right]\left\{\begin{array}{ll}
A_{f f}^{T} & U_{f} \\
A_{s s}^{T} & U_{s}
\end{array}\right\}
$$

where

$$
G=-L_{f s}^{\prime} L_{s s}^{\prime}-1
$$


the companion pressure (compatibility) constraint expression is

$$
\left\{\begin{array}{l}
P_{f} \\
P_{s}
\end{array}\right\}=\left[\begin{array}{ll}
I & 0 \\
G & I
\end{array}\right]\left\{\begin{array}{l}
P_{f} \\
P_{s}^{\prime}
\end{array}\right\}
$$

Physically the matrix G must consist of a row matrix with unit entries when $P_{f}$ represents a single reference pressure. It is noted now that the use of an identity matrix in equation (28) and equation (30), implying that $P_{f}$ and $U_{f}$ may comprise pressure and acceleration subsets rather than individual quantities, is deliberate; the case in which a free surface pressure subset is null is therefore covered by the subsequent development.

Substitution of equations (29) and (30) into equation (22a) yields

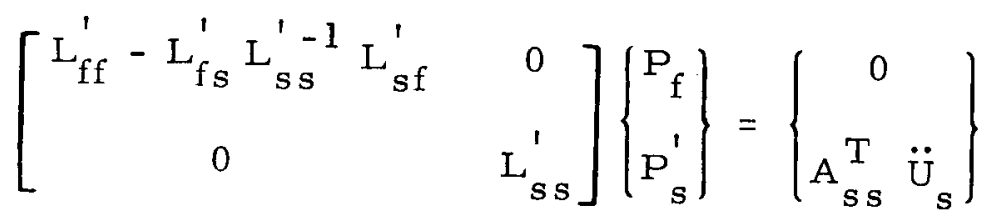

The lower partition merely represents the result already obtained in equation (27b). The upper partition may be interpreted in two ways which consists of (1) the general case in which a single reference pressure, $P_{f}$, is chosen to express the uniform pressure state and (2) the special case in which a set of free surface pressures, $P_{f}$, must be zero. For the first general case, the singularity condition is expressed as

$$
L_{f f}^{\prime}-L_{f s}^{\prime} L_{s s}^{\prime}-1 L_{s f}=0
$$

The constraints presented in equations (29) and (30) are now applied to the structural dynamic equation set equation (20), noting equations (28) and (31), resulting in the symmetric kinematic equation set

$$
\left(M_{s}^{*}+M_{f}\right) \ddot{U}_{s}+K_{s}^{*} U_{s}=0
$$

where the fluid mass matrix is

$$
M_{f}=A_{s s} L_{s s}^{\prime-1} A_{s s}^{T}
$$


and the constrained structural mass and stiffness matrices are

$$
M_{s}^{*}=\Gamma^{T} M_{f f} \Gamma+\Gamma^{T} M_{f s}+M_{s f} \Gamma+M_{s s}
$$

and

$$
\mathrm{K}_{s}^{*}=\Gamma^{\mathrm{T}} \mathrm{K}_{\mathrm{ff}} \Gamma+\Gamma^{\mathrm{T}} \mathrm{K}_{\mathrm{fs}}+\mathrm{K}_{\mathrm{sf}} \Gamma+\mathrm{K}_{\mathrm{ss}}
$$

Complete displacement recovery is obtained through equation (28b) and pressure deviation recovery is obtained through equation (28a). Surface pressure recovery is achieved by combining the upper partition equation set in equation (20) with equation (28b); thus the surface pressure recovery equation set consists of

$$
P_{f}=A_{f f}^{-1}\left(M_{f f} \Gamma+M_{f s}\right) \ddot{U}_{s}+A_{f f}^{-1}\left(K_{f f} \Gamma+K_{f s}\right) U_{s}
$$

and equations (28a) and (30).

The symmetric kinematic formulation developed above is useful in hydroelastic analyses for which either a fluid free surface is not present or free surface gravitational potential and/or ullage stiffness is significant. In most practical analyses involving tanks partially filled with fluid, the free surface strain energy is insignificant relative to the structural energy. In such cases low frequency slosh dynamics is approximated with rigid structure and flexible structure/fluid interaction dynamics is approximated with zero free surface pressure. In the latter case, the reference pressure set, $P_{f}$, consists of all free surface pressures (set to zero) and the reference surface displacement set $U_{f}$ consists of all free surface displacements. It is noted again as in the opening of this section that when a free surface is present $M_{f f}$ and $M_{f s}$ are null; and when surface strain energy is insignificant, $K_{f f}$ and $K_{f s}$ are null.

\section{DISPLACEMENT SET REDUCTION FOR THE HYDROELASTIC PROBLIM}

\section{General Considerations}

For typical launch vehicle propellant tank models, the structural grid displacement set, $U_{S}$, may be fairly large (in excess of 1000 degrees of freedom) and the fluid mass matrix, equation $(32 \mathrm{~b})$, is typically full. Reduction to a much smaller dynamic set of variables is therefore very desirable for computational economy in free vibration analysis. The fullness of the mass matrix is peculiar to the hydroelastic problem and therefore special care must be taken in the reduction process. 
Consider the reduction transformation

$$
\mathrm{U}_{\mathrm{s}}=\mathrm{T}_{\mathbf{s} \mathrm{d}} \mathrm{U}_{\mathrm{d}}
$$

formed by static condensation of the stiffness matrix (Guyan reduction) or by some other process. Application of this transformation to the hydroelastic equation set, equation (32), with the constituent matrices explicitly defined yields the expressions for reduced mass and stiffness matrices. The most critical operations with respect to computational economy are those used in formation of the reduced fluid mass matrix

$$
\left(\mathrm{M}_{\mathrm{f}}\right)_{\text {reduced }}=\left(\mathrm{T}_{\mathrm{sd}}^{\mathrm{T}} \mathrm{A}_{\mathrm{ss}}\right) \mathrm{L}_{\mathrm{ss}}^{-1}\left(\mathrm{~A}_{\mathrm{ss}}^{\mathrm{T}} \mathrm{T}_{\mathrm{sd}}\right)
$$

If the fluid mass matrix in the structural grid set, equation (32b), is explicitly formed, a symmetric reduction process with $\mathrm{T}_{\mathrm{sd}}$ may be quite time consuming and expensive due to the fullness of the original mass matrix. However, by first forming the reduced generalized area matrix $\mathrm{A}_{\mathrm{S}}^{\mathrm{T}} \mathrm{T}_{\mathrm{s}}$ as implied above in equation (35), the large, full fluid mass matrix need not be explicitly formed; the reduced mass matrix is calculated only, resulting in substantial computational economy。

Harmonic Reduction of Geometrically Axisymmetric Structures

The general category of structures of interest in propellant tank hydroelastic analysis consists of configurations which possess, for the most part, geometrically axisymmetric fluid cavities. The tank structure may have a variety of structural asymmetries such as circumferential thickness variation in both shell and rings, discrete longitudinal stiffeners and asymmetric supports. The finite element description of such a structure typically must have a fine and fairly uniform nodal mesh distribution for adequate description of the structural behavior. The use of Guyan reduction in such cases (when the grid-set displacement degrees of freedom are on the order of thousands) may be inefficient and result in an inaccurate dynamic description when extreme coordinate reduction is used. An alternative scheme, which expresses the circumferential displacement distribution in terms of a chosen set of harmonics (harmonic reduction), alleviates the difficulties encountered with Guyan reduction. This is especially true when only a few harmonic shapes appear sufficient to describe the anticipated dynamic behavior of interest.

Harmonic reduction of a discrete structural grid is accomplished by use of the geometric transformation (see ref. 9 for details)

$$
U_{s}=T_{h} U_{h}
$$


where $U_{s}$ corresponds to the physical grid degrees of freedom to be transformed, and $U_{h}$ corresponds to the harmonic degrees of freedom (plus any discrete degrees of freedom not transformed). The transformation or constraint matrix $\mathrm{T}_{\mathrm{h}}$ is composed of the appropriate sinusoidal functions evaluated at the discrete variable locations. It should be noted that discrete displacements expressed in terms of cylindrical or spherical reference frames are most convenient for this procedure. For a typical shell struc ture with $\mathrm{JxK}$ grid points $\mathrm{such}$ that there are $\mathrm{J}$ meridional rows and $\mathrm{K}$ cir cumferential points in a row, the grid set has typically $6 \times \mathrm{JxK}$ degrees of freedom and the matrix semibandwidths are $6 \times \mathrm{K}$ (assuming $\mathrm{K}<\mathrm{J}$ )。 Application of the harmonic transformation as a reduction scheme, where the number of harmonics $N$ is much less than $K$, results in a $U_{h}$ set of $6 \times \mathrm{JxN}$ generalized coordinates with matrix semibandwidths of $6 \times N^{\text {. }}$. If $\mathrm{N} \ll \mathrm{K}$ harmonic reduction represents a radical reduction in the number of degrees of freedom as well as matrix bandwidth. Further reduction of the system description is possible by a small Guyan reduction made by choosing the generalized rotation degrees of freedom and tangential degrees of freedom as members of the omitted set of displacements. In such a case the analysis set consists of $\mathrm{JxN}$ degrees of freedom. This represents a radical reduction in degrees of freedom by a factor of $(\mathrm{N} / 6 \mathrm{~K})$, without a costly large matrix decomposition typical of Guyan reduction.

\section{NUMERICAL STUDIES}

The new incompressible hydroelastic formulation and harmonic re duction have been implemented in NASTRAN and verified and demonstrated on a number of problems. The problems fall into two categories, namely, analytical verification problems for which exact solutions are known, and demonstration problems for which experimental data are available. The 1/8-scale Space Shuttle external tank is included in the second category. The fluid idealizations utilized in the hydroelastic problems are based on existing elements of revolution in NASTRAN.

The present hydroelastic analysis employs NASTRAN structural as well as fluid elements and provides for a description of dynamics of axisymmetrically configured fluids in terms of circumferential harmonic pres sure distributions. The distribution of pressure is typically

$$
\begin{aligned}
p\left(r_{i}, \theta_{i}, z_{i}\right)= & p_{o}\left(r_{i}, z_{i}\right)+\sum_{k=1}^{N}\left\{p_{k}\left(r_{i}, z_{i}\right) \cos k \theta_{i}\right. \\
& \left.+p_{k}^{*}\left(r_{i}, z_{i}\right) \sin k \theta_{i}\right\}
\end{aligned}
$$


The fluid containing structure is described in terms of discrete physical displacements so that the structural representation is not limited to structurally axisymmetric containers. Coupling of harmonic pressure distributions with discrete structural displacements in this formulation is not strictly consistent; moreover, in many cases it is inefficient. When a structure described in terms of discrete displacements is coupled with a fluid described in terms of circumferential harmonics, inconsistencies may arise if too few pressure harmonics are utilized; structural deformation shapes associated with higher harmonics not included in the fluid representation will reflect a lack of fluid inertia loading. Alternatively, when the discrete structural grid is too coarse to accurately describe the highest harmonic pressure dis tributions, large errors in the mode shapes associated with higher harmonics will be present. A consistent grid representation is realized by use of harmonic reduction when the number of structural harmonics coincides with the number of fluid pressure harmonics. This provides additional motivation for the use of harmonic reduction which is peculiar to use of the NASTRAN fluid elements. When harmonic reduction is not utilized, special care must be taken to utilize fluid harmonic and discrete structural descriptions of equivalent complexity.

\section{Analytical Verification Problems}

Spherical Cap. Harmonic reduction was first demonstrated on a spherical cap structure of uniform thickness to radius ratio, $\mathrm{h} / \mathrm{R}=0.05$. The base of the cap, 60 degrees from the pole, is taken as rigidly fixed. The material properties are: elastic modulus $E=107$, a Poisson's ratio $\nu=0.3$, and mass density $\rho_{s}=0.05$.

A nodal grid, consisting of 20 circumferential divisions in a semicircle and 10 meridional divisions, was chosen resulting in a structural model with 1266 DOF (degrees of freedom). Three circumferential harmonics $(0,1,2)$ were chosen for harmonic reduction. The apex node was left in terms of rectangular coordinates since the polar degrees of freedom have no meaning at this node. After application of the fixed-base boundary condition and symmetric kinematic constraints at the pole, and a small Guyan reduction, an analysis set of 72 outward-normal and meridional generalized harmonic displacements resulted. At this point, all natural frequencies and the first 15 modes were calculated. Circumferential harmonics were uncoupled because of the axisymmetry in shell thickness.

The results of the above approach were compared to results based on various Guyan reduction strategies and to "exact" results based on the STARS-II program (ref. 11). A comparison of computed natural frequencies ( table I) indicate that the overall accuracy of the $72-$ DOF harmonic reduction representation is better than the 190-DOF Guyan reduction representation. The computation time associated with eigenvalue analysis of the harmonic analysis set is much less than that associated with the Guyan reduction analysis set. Central processing unit (CPU) times were 238 and 531 seconds, for the harmonic and Guyan reduction representations, respectively. This is 
attributed to elimination of a large-scale matrix decomposition, characteristic of Guyan reduction, and to the fact that much fewer degrees of freedom are required for comparable accuracy (e.go, harmonic 72 -DOF for 5\% accuracy versus Guyan $190-D O F$ for $12 \%$ accuracy.

Fluid-Filled Hemisphere. The first problem for hydroelastic verification consists of an open hemispherical container filled with fluid. The container is massless and follows the artificial structural law

$$
\mathrm{p}=a \mathrm{u}_{\mathrm{r}}
$$

where $u_{r}$ is the local $r$ adial displacement; the exact free-vibration solution is known (refo 12). The finite element model of the fluid and container is illustrated in figure 1. A diagonal structural stiffness matrix with entries

$$
\mathrm{K}_{\mathrm{ii}}=a \mathrm{~A}_{\mathrm{i}}
$$

results from the artificial structural law in which $A_{i}$ is the area associated with the "ith" radial degree of freedom. The fluid model is expressed in terms of the circumferential pressure harmonics $\mathrm{n}=0,2,4$ and the structural surface and free surface grids are reduced by harmonic reduction accordingly. The fluid mass matrix is expressed in terms of a 21-DOF analysis set of structural radial displacements at seven meridional locations.

A comparison of exact and finite element nondimensional natural frequencies is presented in table II and comparisons of selected modal dis placement distributions are presented in figure 2. The finite element results are in very good agreement with the exact solution, with the level of accuracy decreasing with modal complexity as expected.

Fluid-Filled' Cylinder. Another hydroelastic verification problem consists of the fluid-filled, circular cylindrical shell illustrated in figure 3. The shell structure is taken as one with bending as well as membrane stiffness. The geometric properties of the shell consist of a cylinder with length $/$ radius ratio $(\ell / R)=2$ and a thickness $/ \mathrm{radius}$ ratio $(\mathrm{h} / \mathrm{R})=0.01$. In addition, the ratio of fluid to structure density $\left(\rho_{f} / \rho_{s}\right)=1 / 3$ and the structural material Poisson ratio $(v)=0.3$. An exact hydroelastic modal solution is known for an infinitely long cylinder (refs. 13, 14) which holds for the present problem when the structure is subjected to the boundary conditions

$$
\begin{gathered}
u_{r}=M_{\theta}=N_{\theta}=N_{z}=0 \begin{array}{c}
\text { (shear diaphragm for } \\
z=\ell, r=R)
\end{array} \\
p=0 \text { (free surface) for } z=\ell, r \leq R
\end{gathered}
$$




$$
\begin{gathered}
\frac{\partial u_{r}}{\partial z}=u_{z}=\frac{\partial M_{z}}{\partial z}=N_{z}=0 \begin{array}{c}
(\text { symmetry) for } \\
z=0, r=R
\end{array} \\
u_{z}=0 \text { (fixed bottom) for } z=0, r \leq R
\end{gathered}
$$

The finite element models of the quarter shell $(-0 \leq z \leq \ell, 0 \leq \theta \leq$ $90 \mathrm{deg}$ ) and fluid were constructed taking advantage of symmetry. The structural grid for the quarter shell consists of 726 DOF ( 11 meridional nodal rows, 11 circumferential nodal columns) and the fluid grid consists of 165 DOF (55 nodes of rotation, circumferential harmonics $n=0,2,4$ ). A 30-DOF set was obtained using (1) a harmonic transformation retaining harmonics $n=0,2,4$, (2) the application of single-point constraints to enforce boundary conditions, and (3) a small Guyan reduction retaining only radial dis placements.

All 30 natural frequencies and 25 mode shapes with and without the fluid included were calculated. Frequency spectra for the empty and fluidfilled shells are presented in figure 4, illustrating generally excellent comparison between finite element and exact results in both cases.

A characteristic of the present formulation, which is as significant as numerical accuracy, is computational economy. On the IBM $370 / 165 \mathrm{com}$ pute $r$ the total solution time for the empty cylinder was about 2 CPU minutes; for the fluid-filled cylinder an additional CPU minute was required to form the fluid mass and pressure recovery matrices.

\section{Comparisons with Experimental Data}

Liquid-Filled Cylinders Under Static Pressurization. A detailed experimental study of the dynamics of structurally axisymmetric and as ymmetric circular cylinders under various water fill and static pressurization conditions has been conducted at NASA Langley Research Center by Mr. Robert Herr. Data resulting from these tests (unpublished) are quite complete and provide an excellent basis for analysis/test correlation studies。 The test articles are aluminum cylinders with mean radius of $25.4 \mathrm{~cm}$ (10 in.) and height of $50.8 \mathrm{~cm}(20 \mathrm{in}$. ). The cylinder walls are welded at the top and bottom to heavy aluminum plates. The axisymmetric test article has a cylinder wall thickness of $0.081 \mathrm{~cm}(0.032 \mathrm{in}$.) and the asymmetric test article has a wall thickness variation around the circumference of 0.051 $0.102 \mathrm{~cm}(0.020-0.040 \mathrm{in.})$ according to the equation

$$
\frac{h}{h_{\max .}}=0.75+0.25 \cos \theta
$$


A structural model for the half-cylinder $(0 \mathrm{deg} \leq \theta \leq 180 \mathrm{deg})$ taking advantage of the single axis of symmetry was assembled with sufficiently fine grid to simulate the circumferential harmonic shapes up to $\mathrm{n}=15$, which were known a priori from the experimental results to dominate in the lowest frequency modes. The structural grid set consists of 2046 DOF resulting from 11 axial grid rows and 31 circumferential grid columns all evenly dis tributed. The fluid representation chosen at the half-filled condition is illustrated in figure 5. The 480-DOF (pressures) fluid model results from the 30 fluid-grid locations of revolution expressed in terms of the circumferential harmonics $\mathrm{n}=0$ to 15 , with a sufficiently fine grid near the structural wall to simulate the sharp pressure gradients occurring in the higher harmonics. Since modes with significant harmonic content up to $\mathrm{n}=15$ were of interest, harmonic reduction was not utilized here; computatiunal economy would not be improved by the harmonic transformation and thus only a Guyan reduction was utilized.

A series of cases including symmetric and asymmetric cylinders in the half-filled and empty configurations were studied. In addition, the effects of static pressurization were included by use of differential stiffness capability in NASTRAN.

The first cases studied pertained to the cylinder of uniform thickness. The empty cylinder was first considered with an assumed axial plane of symmetry at $\mathrm{z}=25.4 \mathrm{~cm}$ such that only $\mathrm{m}=1,3,5$ modes would be calculated. The grid set of $1116 \mathrm{DOF}$, consisting of nodes below $\mathrm{z}=25.4 \mathrm{~cm}$, was reduced by Guyan reduction to an analysis set of 276 radial DOF with the lower end completely fixed (clamped). The unpressurized and pressurized shell frequency spectra of $m=1, n \geq 4$ modes are illustrated in figure 6 along with the test results. The calculated frequency spectrum was higher than the experimental frequency spectrum for both unpressurized and pressurized conditions. A series of modifications of the structural model to reconcile the differences in results were considered. It was finally concluded that axial flexibility idealized as an axially free condition in the cylinder/plate weld, provided the proper correction. Incorporation of the relaxed boundary conditions

$$
u_{r}=\frac{\partial u_{r}}{\partial z}=N_{z}=0 \quad \text { for } z=0,50.8 \mathrm{~cm}
$$

resulted in extremely accurate frequency spectra for the empty cylinder as illustrated in figure 6 .

The half-filled condition was then considered. The cylinder structure in this case does not have a dynamic plane of symmetry at $z=25.4 \mathrm{~cm}$; the lower portion $(z \leq 25.4 \mathrm{~cm})$ is loaded by fluid structural inertia whereas the upper portion $(z>25.4 \mathrm{~cm})$ is loaded only by the structural inertia. This provides motivation for Guyan reduction with all degrees of freedom at and above $\mathrm{z}=25.4 \mathrm{~cm}$ omitted (not including the supported degrees of freedom). A Guyan reduction on the structure and fluid was then performed resulting in an analysis set consisting of 248 radial DOF consistent with the fluid mass matrix. Hydroelastic modes, based on the clamped and modified-clamped 
end conditions [eq. (40)], were then calculated. The $\mathrm{m}=1, \mathrm{n} \geq 4$ modal frequency spectra, illustrated in figure 6 , show that the representation with the relaxed end conditions is quite accurate, as concluded in the empty case.

The unsymmetric cylinder structural model consists of the same grid set as in the case of the axisymmetric cylinder, and the Guyan reduction discussed above was utilized. The hydroelastic study of this cylinder was limited to the half-filled condition with the "realistic"edge conditicn applied. The $m=1$ mode shapes illustrated in figure 7 are in very good agreement with the unpressurized and pressurized test results, as are the modal frequencies.

Computation times for the cylinder study were moderate since harmonic reduction, which was not appropriate, was not utilized. In all cases considered, all 248 eigenvalues and 25 eigervectors were calculated.Computation time for the empty axisymmetric cylinder was 509 CPU sec. Preparation of fluid matrix data required $97 \mathrm{CPU}$ sec and computation of hydroelastic modes required 1,193 CPU sec. The increased CPU time required in this case is predominantly due to the increased structural grid set size of the fluid-filled cases; the increase in Guyan reduction time for systems of equivalent matrix bandwidth is proportional to the increase in grid set degrees of freedom. Computation times for the unsymmetric cylinder were similar to those required for the axisymmetric cylinder.

The 1/8-Scale Space Shuttle External Tank. An investigation of the dynamics of a l/8-scale Space Shuttle external tank in a free-free supported condition is in progress at NASA Langley Research Center. The 1/8-scale external tank consists of two separate propellant tanks connected by a cylindrical section. Although the fluid/structure interface is axisymmetric, the tank structure contains thickness and stiffener as ymmetries. The finite element hydroelastic model for half the structure, taking advantage of the single axis of symmetry, is described in detail in references 9 and 15. It consists of a grid set of 348 pressure DOF and 2058 structural DOF (and 768 harmonic structural DOF to be used in harmonic reduction). The structural grid deformed in the fundamental bending mode is illustrated in figure 8. Harmonics $\mathrm{n}=0,1,2,3$ were chosen to describe asymmetric dynamics with the pitch plane taken as an axis of symmetry. The analysis set of displacements resulting from a combination of harmonic and Guyan reductions consists of 128 harmonic DOF associated with outward normal motion of the tank wall.

Three conditions have been studied consisting of nearly full, intermediate and empty propellant fill conditions. For each of the fill conditions, 128 natural frequencies and 25 mode shapes and modal pressure distributions were calculated with very good computational efficiency. About 20 CPU minutes per liquid level on the IBM $370 / 165$ computer was required to perform the entire analysis including matrix assembly, reduction and modal analysis. In previous attempts to study the dynamics of the same finite element representation with the standard unsymmetric NASTRAN hydroelas tic analysis, computation times were about 52 CPU minutes for only one natural frequency and mode shape from a $412-$ DOF analysis set (ref. 15). 
Excellent agreement between analysis and experimental frequencies occurred in the first axial mode but poor agreement occurred in the bending modes. A thorough investigation of the fluid model revealed good consis tency in the idealization. As a result, the source of the discrepancy is believed to be in the finite element representation of the structure which was constructed prior to the present work。

\section{CONCLUDING REMARKS}

Symmetric finite element matrix formulations for compressible and incompressible hydroelasticity have been developed on the basis of Toupin's complementary formulation of classical mechanics. The incompressible formulation applicable in propellant tank hydroelastic analysis has been implemented in NASTRAN to replace an inefficient unsymmetric matrix formulation. The new technique which utilizes existing fluid and structural finite elements has been verified and demonstrated to be accurate and efficient.

The fluid representation in the incompressible case reduces to a symmetric fluid mass matrix described in terms of surface deformation only upon recognition of a singularity in the fluid inertance matrix. The singularity describes a physically necessary compatibility condition in that it assures that the incompressible idealization will not move under uniform pressure. Moreover, the singularity defines a kinematic constraint which is applied to the structural idealization when the fluid is completely bounded by a structural interface and when free surface ullage and/or gravitational stiffness are significant. The fluid mass matrix is added directly to the structural mass matrix, forming a symmetric set of hydroelastic equations in terms of structural displacements. Modal hydroelastic analysis is performed with the same efficiency as in the case of a non-fluid-filled structure, since no additional degrees of freedom are required for the fluid (other than free surface displacements when necessary).

The efficiency of the new hydroelastic analysis technique has been enhanced for both fluid and structure by introduction of harmonic reduction, applicable to geometrically axisymmetric structures, as an alternative to Guyan reduction. When the number of harmonics utilized is much less than the number of discrete nodes about a circumference, overall matrix size and bandwidth are significantly reduced.

The formulation has been verified by comparison with exact analytical results for a fluid-filled hemispherical container and a fluid-filled circular cylindrical shell。 In all cases, excellent correlation was exhibited as well as very good computational efficiency. In addition, the analysis/test correlation study on symmetric and unsymmetric circular cylindrical shells under various fluid-fill conditions is considered very good.

Analysis/test discrepancies on the 1/8-scale external tank model for the space shuttle have not yet been resolved. The efficiency of the current analysis, however, is very encouraging based upon comparison of 
computation times between the present and the standard unsymmetric NASTRAN hydroelastic formulations.

The more general kinetic formulation which includes fluid compressibility has yet to be investigated in detail and applied. Typical applications include underwater explosion and acoustic analysis in general. In addition, a set of polyhedral complementary fluid finite elements should be incorporated to allow for modeling of general fluid configurations.

\section{REFERENंCES}

1. Prevention of Coupled Structure-Propulsion Instability (POGO). NASA SP-8055, Oct. 1970。

2. Pinson, L. D. and Brown, C. G. : A Finite Element Method for Nonaxisymmetric Vibrations of Pressurized Shells of Revolution Par tially Filled with Liquid. AIAA Paper No.73-399, Mar. 1973.

3. Feng, G. C。 and Jones, C。E。: Dynamics of a Flexible Bulkhead and Contained Fluid。 Lockheed Missiles and Space Co., Huntsville Res. and Eng。Center, Final Report LMSC-HREC TR D306476, May 1973.

4. Palmer, J。H. and Asher, G. W. : Calculation of Axis ymmetric Longitudinal Modes for Fluid-Elastic Tank-Ullage Gas Systems and Comparison with Model Test Results. AIAA Symposium on Structural Dynamics and Aeroelasticity, Aug. 30-Sept. 1, 1965, pp. $189-$ 193.

5. Guyan, R. J.: Study on Dynamics of an Ellipsoidal Bulkhead Containing Fluid. Rockwell International, Space Div., Tech. Summary Report SD71-183, Sept. 1971.

6. Goldman, R。L.: Longitudinal Vibration Analysis of Partially Filled Ellipsoidal Tanks by Finite Differences。RIAS Tech。Report No. TR70-6C, Aug. 1970.

7. MacNeal, R.H. (Ed.): The NASTRAN Theoretical Manual - Addendum: Chapter 16, Interaction of Structures and Fluids. NASA SP-211, Apr. 1, 1972.

8. Toupin, R. A. : A Variational Principle for the Mesh-Type Analysis of a Mechanical System. J. Appl. Mech。, June 1952, pp. 151-152.

9. Coppolino, R. N.: A Numerically Efficient Finite Element Hydroelastic Analysis. Vol. I: NASA CR -2662, Apr. 1976, Vol. II: NASA CR 132684 , Nov. 1974.

10. Crandall, S. No, et a1: Dynamics of Mechanical and Electromechanical Systems, McGraw-Hill Book Co。, 1968. 
11. Svalbonas, V. and Ogilvie, P.: Numerical Analysis of Stiffened Shells of Revolution. NASA CR-2273, Sept. 1973.

12. Coppolino, R. N.: Exact Hydroelastic Solution for an Ideal Fluid in a Hemispherical Container. J. Spacecraft and Rockets, vol. 10, no. 9, Sept. 1973, pp. 612-613.

13.. Abramson, H. N. (Ed. ): The Dynamic Behavior of Liquids in Moving Containers. NASA SP-106, 1966, pp. 321-338.

14. Leissa, A. W.: Vibration of Shells. NASA SP-288, 1973, pp. 62-64, 248-265.

15. Bernstein, M., et al: Development of Technology for Fluid-Structure Interaction Modeling of a 1/8-Scale Dynamic Model of the Shuttle External Tank (ET). NASA CR-132549, Aug. 1974. 

TABLE I. - 60 -DEG SPHERICAL CAP - COMPARISON OF
MODAL FREQUENCIES

\begin{tabular}{|c|c|c|c|c|}
\hline \multicolumn{2}{|c|}{ Mode } & "Exact" & Harm. Red. (72 DOF $)$ & Guyan Red. (190 DOF) \\
\hline $\mathrm{m}$ & $\mathrm{n}$ & $\Omega$ exact & Error* & Error \\
\hline 1 & 0 & 637 & 0.015 & 0.029 \\
\hline 2 & & 697 & 0.004 & 0.020 \\
\hline 3 & & 720 & 0.016 & 0.051 \\
\hline 4 & & 760 & 0.023 & $\ldots$ \\
\hline 5 & & 820 & 0.028 & $\ldots \ldots$ \\
\hline 1 & 1 & 611 & 0.008 & 0.047 \\
\hline 2 & & 687 & 0.012 & 0.037 \\
\hline 3 & & 715 & 0.015 & 0.045 \\
\hline 4 & & 738 & 0.029 & 0.074 \\
\hline 5 & & 810 & 0.0 & 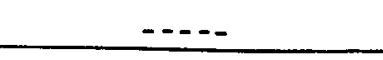 \\
\hline 1 & 2 & 684 & 0.023 & 0.067 \\
\hline 2 & & 711 & 0.029 & 0.075 \\
\hline 3 & & 742 & 0.027 & 0.084 \\
\hline 4 & & 765 & 0.060 & $-\cdots$ \\
\hline 5 & & 831 & 0.036 & 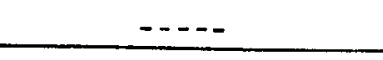 \\
\hline & 3 & 694 & $\cdots$ & 0.088 \\
\hline 2 & & 714 & $-\ldots$ & 0.103 \\
\hline
\end{tabular}

*Error $=\frac{\Omega}{\Omega \text { exact }}-1$

TABLE II. - FLUID IN A HEMISPHERICAL CONTAINER NATURAL FREQUENCY COMPARISONS

\begin{tabular}{|c|c|c|c|c|}
\hline $\mathrm{m}$ & $\Omega_{\text {exact }}{ }^{*}$ & & Error ${ }^{* * *}$ & \\
\hline & & $n=1$ & $n=2$ & $n=4$ \\
\hline 1 & 1 & 0 & & \\
\hline 3 & 1.732 & -0.02 & 0.089 & \\
\hline 5 & 2.236 & -0.02 & 0.059 & 0.032 \\
\hline 7 & 2.646 & -0.065 & 0.026 & 0.020 \\
\hline 9 & 3. & -0.042 & -0.003 & -0.052 \\
\hline 11 & 3.317 & -0.103 & -0.014 & -0.038 \\
\hline 13 & 3.606 & -0.168 & 0.071 & -0.073 \\
\hline \multicolumn{5}{|c|}{$*_{\Omega_{\text {exact }}}=\sqrt{\mathrm{m}} \mathrm{m}, \mathrm{ODD}>\mathrm{n}$ (see Ref. 12 for details) } \\
\hline \multicolumn{5}{|c|}{ Error $=\frac{\Omega}{\Omega}-1$} \\
\hline
\end{tabular}


FLUID GRID OF REVOLUTION STRUCTURAL GRID FOR QUARTER HEMISPHERE

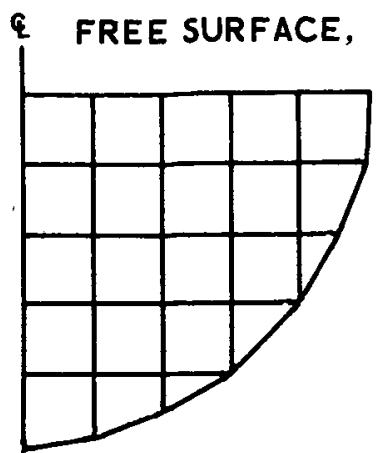

FREE SURFACE, $K_{\mathrm{ii}}=0$

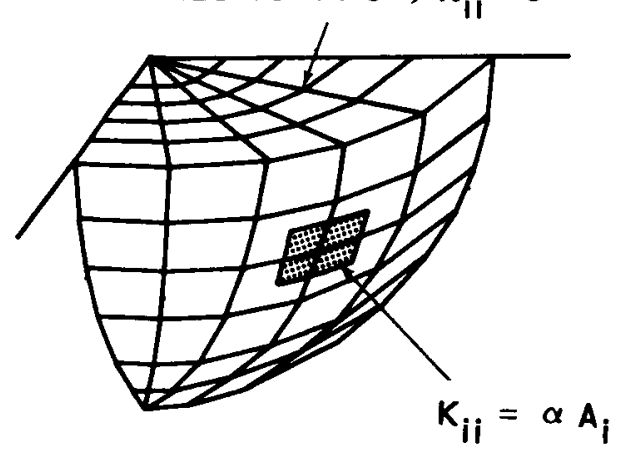

FIGURE 1. - FLUID AND HEMISPHER ICAL CONTAINER MODEL

$\mathrm{m}, \mathrm{n}=1,0$

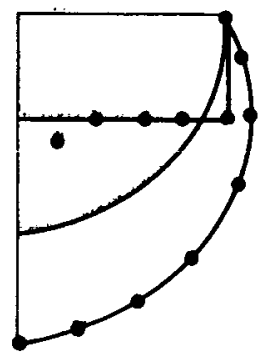

$\mathrm{m}, \mathrm{n}=\mathbf{3 , 0}$

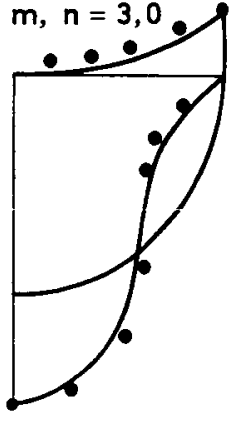

$m, n=5,2$

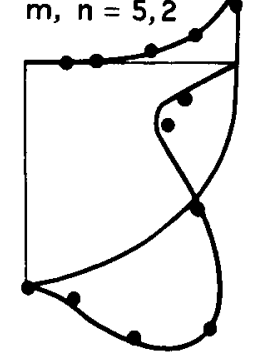

NOTE: DENOTES EXACT MODE SHAPE

- deNOTES FINITE ELEMENT MODE SHAPE $m, n=5,0$

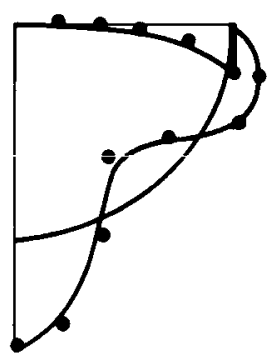

$\mathrm{m}, \mathrm{n}=3,2$

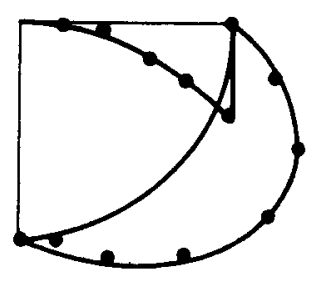

$m, n=5,4$

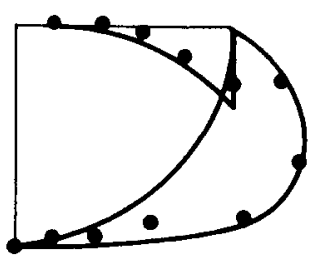

FIGURE 2. - HEMISPHERE HYDROELASTIC MODE SHAPES 


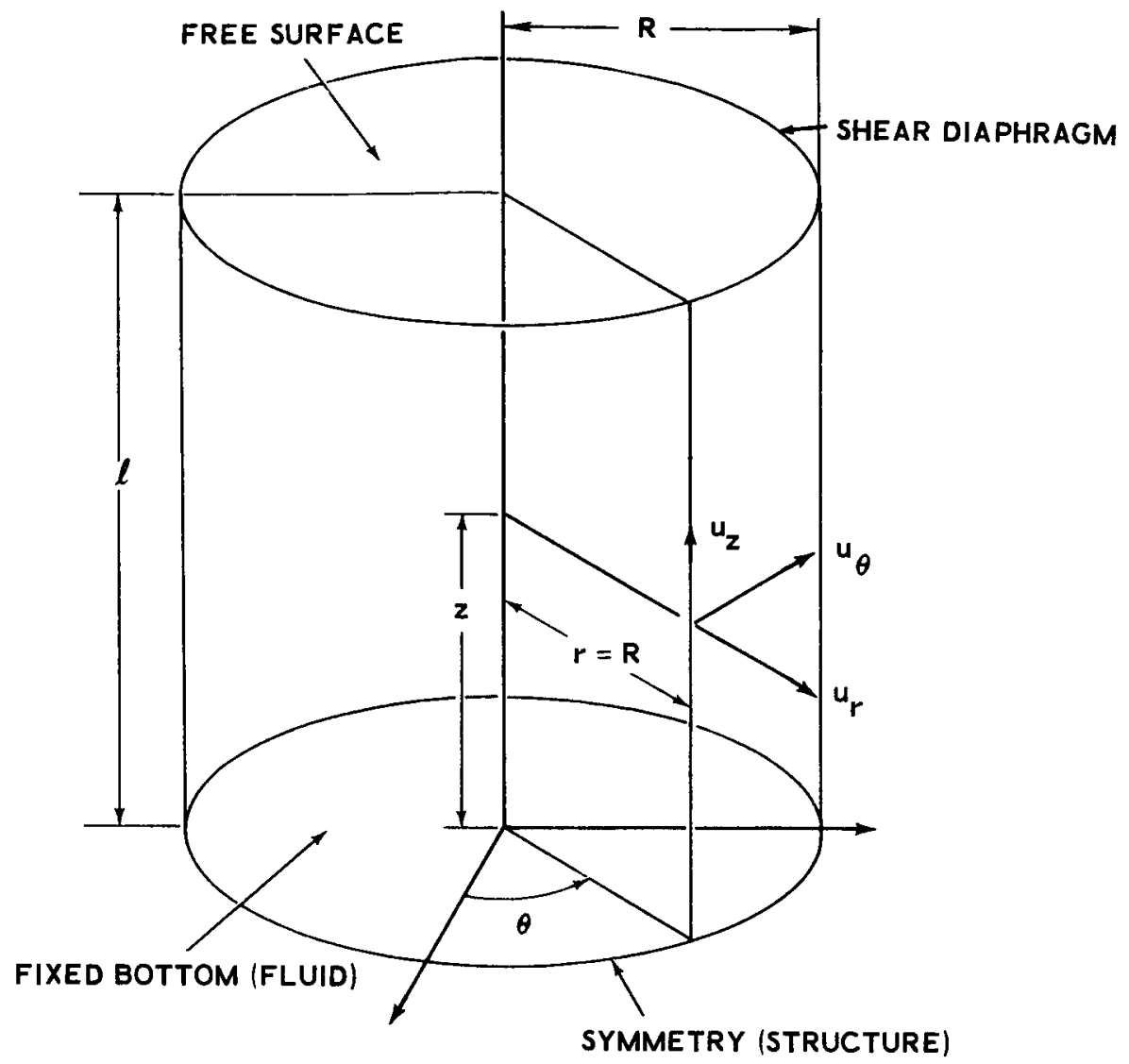

FIGURE 3. - FLUID-FILLED CIR CULAR CYLINDRICAL SHELL
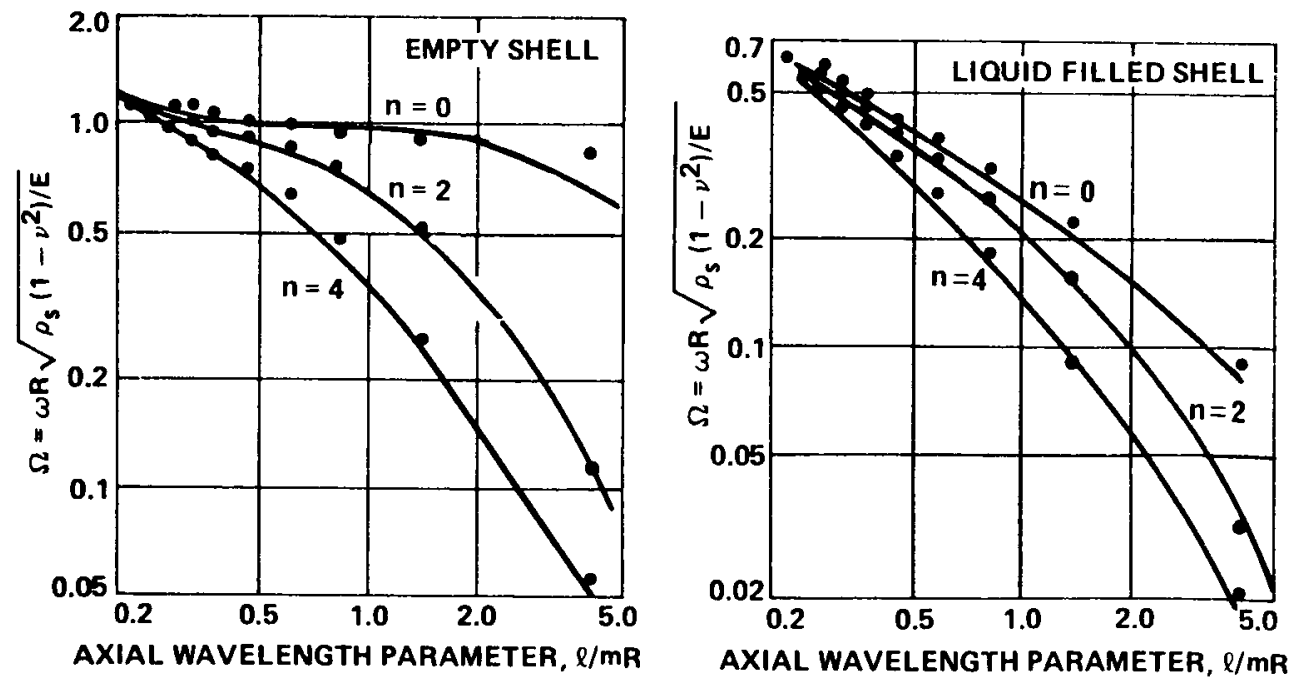

- "EXACT" THEORY

- FINITE ELEMENT SOLN

FIGURE 4. - CIR CULAR CYLINDRICAL SHELL FREQUENCY SPECTRA 


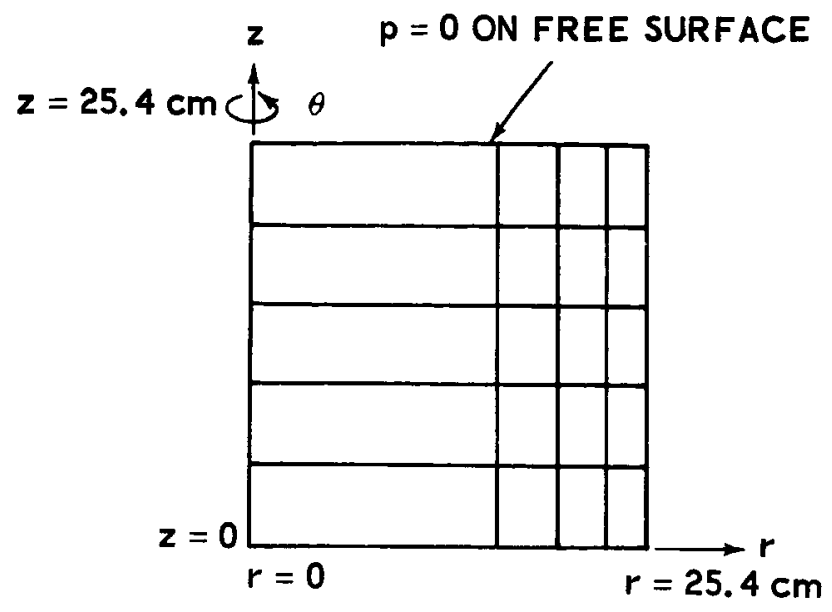

FIGURE 5. - HALF-FILLED CYLINDER FLUID IDEALIZATION
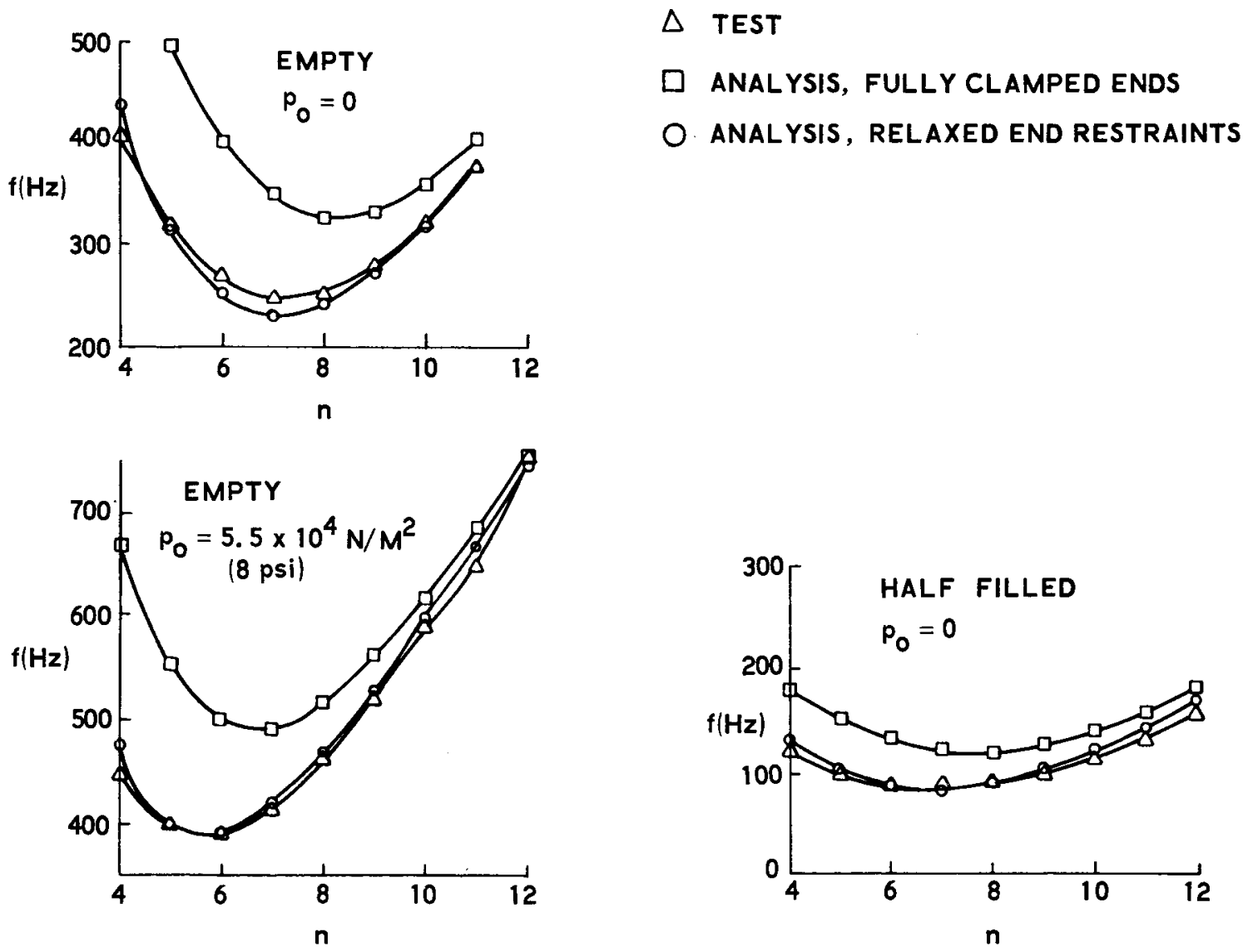

FIGURE 6. - AXISYMMETRIC CYLINDER FREQUENCY SPECTRA ( $\mathrm{m}=1 \mathrm{MODES})$ 

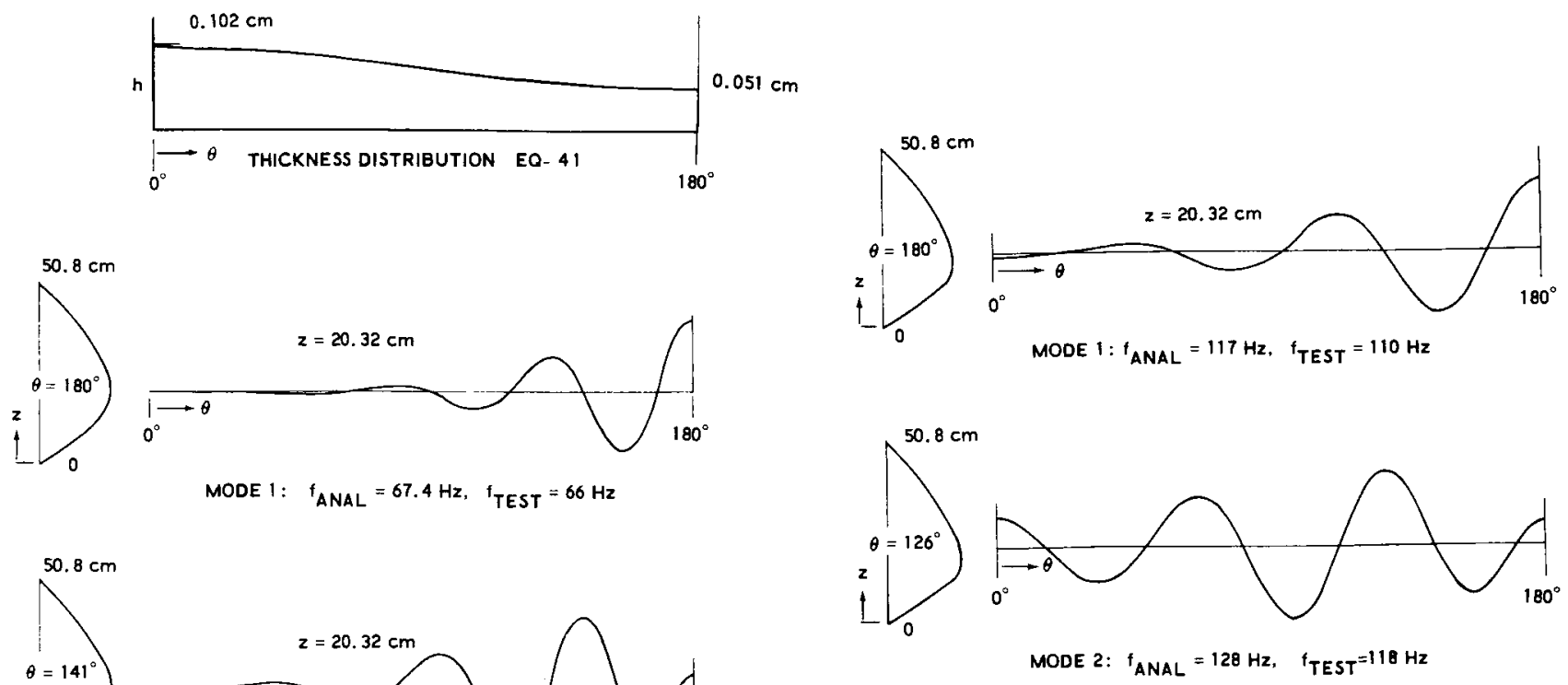

(b) WITH PRESSURIZATION, $p_{0}=5.5 \times 10^{4} \mathrm{~N} / \mathrm{M}^{2}$

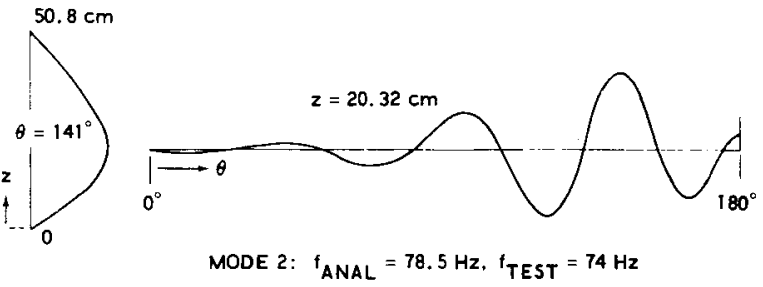

(a) NO PRESSURIZATION, $P_{0}=0$

FIGURE 7。 - UNSYMMETRIC CYLINDER HYDROELASTIC MODES

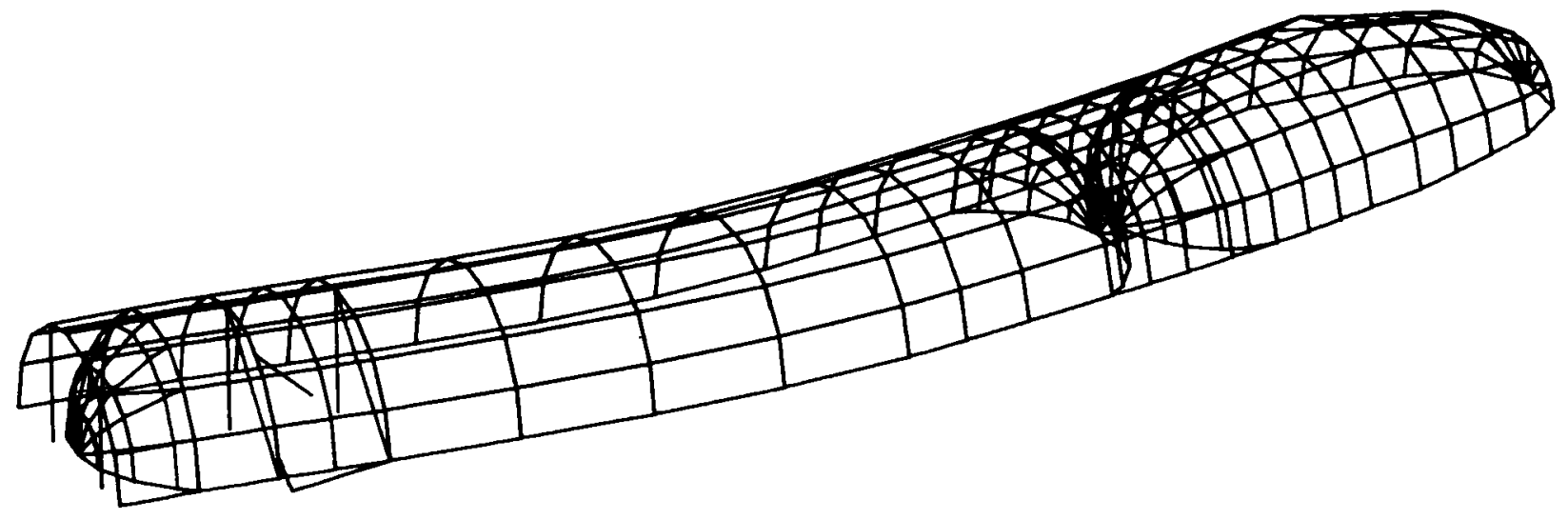

FIGURE 8. - 1/8-SCALE SPACE SHUTTLE EXTER NAL TANK FUNDAMENTAL HYDROELASTIC BENDING MODE 